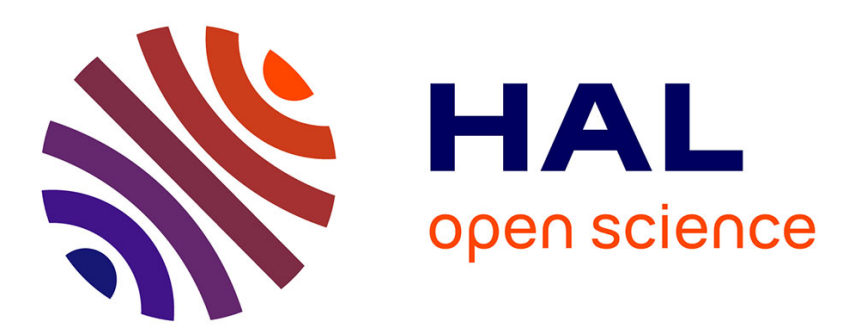

\title{
La dialectique du singulier et du social dans les processus de singularisation: style(s), idiolecte, ethos
}

Alain Rabatel

\section{To cite this version:}

Alain Rabatel. La dialectique du singulier et du social dans les processus de singularisation: style(s), idiolecte, ethos. Pratiques: linguistique, littérature, didactique, 2007, 135-136, pp.15-34. halshs00367541

\section{HAL Id: halshs-00367541 \\ https://shs.hal.science/halshs-00367541}

Submitted on 22 Nov 2009

HAL is a multi-disciplinary open access archive for the deposit and dissemination of scientific research documents, whether they are published or not. The documents may come from teaching and research institutions in France or abroad, or from public or private research centers.
L'archive ouverte pluridisciplinaire HAL, est destinée au dépôt et à la diffusion de documents scientifiques de niveau recherche, publiés ou non, émanant des établissements d'enseignement et de recherche français ou étrangers, des laboratoires publics ou privés. 


\title{
La dialectique du singulier et du social dans les processus de singularisation : style(s), idiolecte, ethos
}

\author{
Alain Rabatel
}

ICAR, UMR 5191, CNRS, Universités de Lyon 2 et Lyon 1

Ainsi que le rappelle opportunément Latour dans un dialogue imaginaire avec un apprenti-chercheur, on peut avoir plusieurs points de vue sur un objet. Loin de conduire au relativisme, l'articulation des points de vue théoriques permet de mieux rendre compte de la complexité objective des objets :

(1) Qu'est-ce qui vous fait penser qu' « adopter un point de vue» signifie « être limité »? ou être spécialement « subjectif »? [...] Si vous pouvez avoir différents points de vue sur une statue, c'est parce que la statue elle-même est en trois dimensions et vous permet, oui, vous permet de tourner autour. Si une chose rend possible cette multiplicité de points de vue, c'est qu'elle est très complexe, intriquée, bien organisée, et belle, oui, objectivement belle.

[...] Ne croyez pas à toutes ces foutaises sur le fait d'être « limité » à votre propre perspective. Toutes les sciences ont inventé des moyens pour se déplacer d'un point de vue à un autre, d'un cadre de référence à un autre. [...] C'est ce qu'on appelle la relativité. [...] Si je veux être un scientifique et atteindre à l'objectivité, je dois être capable de naviguer d'un cadre de référence à l'autre, d'un point de vue à l'autre. Sans de tels déplacements, je serais limité pour de bon dans mon point de vue étroit. (Latour, $2006: 210-213$ )

Cette remarque méthodologique nous servira de guide dans notre approche d'une notion complexe s'il en est, celle du style, et dans celle de quelques unes de ses relations avec les notions affines d'idiolecte et d'ethos, qui ne seront abordées que secondairement.

À rebours des simplifications critiquées par Latour, Genette 2006 : 96, distingue, à propos de «contemporain » (mais le raisonnement pourrait être étendu à d'autres catégories stylistiques) un "sens factuellement temporel, "absolu" si l'on veut par relation implicite au moment de l'énonciation (contemporain tout court, sous-entendu du locuteur) » et un sens « relatif (contemporain de Louis XI, de Napoléon, de Félix Faure) ». La distinction entre prédicats esthétiques, absolus, et prédicats historiques, relatifs, explique qu'une œuvre qui n'appartient pas à une époque dite «baroque » relève néanmoins du style baroque. Autrement dit, les 
approches historico-stylistique et esthético-stylistique appréhendent tantôt le style comme un absolu, tantôt dans sa dimension historique relative ${ }^{(1)}$. Non seulement il est possible de parler valablement du style sous ces deux entrées, mais elles gagnent à être articulées, y compris avec d'autres cadres d'analyse.

Ce raisonnement peut (et devrait) être appliqué non seulement au style, mais encore à l'idiolecte et à l'ethos, pour s'en tenir ici aux notions qui sont au cœur de notre interrogation. Comme il est hors de question de présenter de façon détaillée, en un article, ces trois notions connexes, on traitera ici de leurs relations à partir de l'hypothèse que style, idiolecte et ethos sont travaillés par une même et forte dialectique, celle de l'individuel et du social ${ }^{(2)}$, dans l'expression de la subjectivité. Les théorisations du style, de l'idiolecte ou de l'ethos se distinguent selon l'attention accordée à cette dialectique et aux formes sous lesquelles elle se manifeste. Ainsi l'approche individuelle de l'ethos privilégiera les traits de caractère (singuliers) tandis qu'une approche plus sensible aux vertus qu'il convient de manifester dans son discours selon les circonstances mettra en avant les mœurs, les vertus partagés par tel auditoire. De même, les spécialistes des registres (sublime, moyen, bas) ou de l'approche générique du style ${ }^{(3)}$ accorderont davantage d'attention aux traits stylistiques en lien avec les circonstances, les publics, les genres, tandis que les tenants de la notion de style d'auteur, des approches herméneutiques ou génétiques du style valoriseront les traits singuliers d'une parole singulière, souvent cantonnée dans le domaine artistique ${ }^{(4)}$. Les théorisations de l'idiolecte n'échappent pas à ces tensions, rapprochant les traits idiolectaux des traits sociolectaux ou idiosyncrasiques, oscillant entre caractéristiques linguistiques permanentes ou ajustées en tenant compte du regard d'autrui. Seule une approche moniste est de nature à rendre de compte de l'ensemble des phénomènes en tension selon une approche continuiste de l'actualisation, ainsi qu' on le verra dans la première partie, consacrée plus spécifiquement à l'approche moniste du style, définissant ce dernier comme recherche de l'affirmation de soi et d'une singularisation de la parole dans le cadre du jeu avec les contraintes socio-culturelles des genres, qui s'imposent aux individus.

Dans une deuxième partie, on s'attachera aux relations entre style, idiolecte et ethos. On définira l'idiolecte comme tension singularisante de soi pris dans la dialectique coénonciative et interactionnelle et l'ethos, comme parole incorporée à des fins argumentatives. Dans les trois cas, l'approche moniste ne repose pas sur une sélection arbitraire de traits mais sur l'ensemble du matériau langagier (et des paramètres paraverbaux), rapporté à des genres, à la dialectique du même et de l'autre ou à des fins argumentatives. Les processus de construction et d'affirmation de soi opèrent ainsi dans des processus de singularisation socialisés de part en part.

(1) $C f$. Vouilloux, 2005 pour une analyse des " prédicats stylistiques ». Vouilloux distingue «prédicats caractérisants ", répondant à la question du «Comment? "- subdivisés en prédicats classifiants (dramatique), évaluatifs (sublime, vulgaire) affectifs (poignant) - et "prédicats déterminatifs », répondant aux questions « De qui, d'où, de quand? " (hugolien, baroque, toscan). Cette classification définit le style moins comme expression d'une parole singulière que comme processus de stylisation secondaire et consciente des discours.

(2) Il va sans dire que le social inclut les dimensions culturelles, esthétiques, idéologiques, etc.

(3) Compte tenu de l'abondance de la matière, la réflexion sur le style est limitée à la question langagière.

(4) Toutefois l'analyse génétique ne se cantonne pas nécessairement à l'étude des manuscrits d'écrivain, comme le montre, ici même, l'article de S. Plane. 


\section{Style ou styles?}

Les entrées «style » des dictionnaires spécialisés soulignent l' « hétérogénéité de la conceptualisation » et le "flou de la catégorisation » (Molinié, 1992: 304). Dans le Gradus, le lecteur ploie sous la liste de qualificatifs (bas, calepin, coupé, épithétique, familier, féminin, homérique, hypotaxique, incisif, indirect, indirect libre, noble, oratoire, périodique, savant, simple, soutenu, sublime, télégraphique) qui ne définissent guère la notion ; il est à peine plus éclairé par la rubrique « stylème » (« ensemble de marques du style personnel ») (Dupriez, 1980:416) car la formulation élude le débat sur la nature des marques, continues ou discontinues. Le lecteur du Dictionnaire de rhétorique est confronté à de semblables empilements de qualificatifs avec les styles sublime, moyen, bas (qui recoupent respectivement les styles asian, rodhien, attique) ou encore abondant vs bref, fleuri vs sec. Mais les qualifications emmêlent de façon pré-théorique les notions de genres, de niveaux, sur un plan souvent normatif.

\subsection{Tensions constitutives de la notion de style}

Nous partirons d'un extrait d'un roman récent qui offre le mérite, à nos yeux, de bien poser la complexité de la notion de style, si l'on en juge par les trois définitions différentes qui en sont données successivement par le même locuteur, alors qu'il n'a pas conscience d'exprimer des points de vue distincts, qui mériteraient d'être articulés :

(4) - Maître Osman m'a montré, à partir des merveilleuses images des maîtres anciens, que tu possèdes un style, m'a-t-il répondu. Il m'a expliqué que ce défaut caché, le style, n' apparaît pas chez un artiste de son propre chef, mais du fait de motivations profondes, enfouies dans sa mémoire. Il m'a aussi appris que ces petits écarts, ces erreurs, ces faiblesses, qui, à une époque, celle de nos maîtres anciens, valaient à leurs auteurs l'opprobre et le mépris et se trouvaient proscrits, refoulés de leur art, vont dorénavant, sous l'influence massive et universelle des peintres d'Europe, gagner peu à peu des lettres de noblesse, comme marques de style, d'originalité. Dorénavant, tous les crétins, soucieux d'étaler avec complaisance leurs ineptes insuffisances, s'attachent à rendre le monde bariolé et fou, un monde brouillé, nettement moins parfait. »

Sa façon de s'écouter, de croire à tout ce qu'il débitait, montrait combien il faisait lui-même partie de ces crétins.

«Maître Osman est-il en mesure d'expliquer pourquoi j'ai toujours, pendant toutes ces années, dessiné les chevaux avec des naseaux parfaitement normaux ?

- C'est à cause des coups qu'il vous a donnés, et grâce à l'amour qu'il vous a prodigué, à vous tous. C'est absurde, mais c'est comme cela : vous êtes, à ses yeux, tous semblables, parce qu'il a été, pour vous tous, un père et un amoureux. Ce qu'il voulait, ce n'est pas que chacun développe son style, mais contribue à créer le style de son atelier. Et cette ombre, tutélaire et menaçante à la fois, vous a conduits à oublier vos instincts propres, vos différences les plus profondément ancrées, dans la mesure où elles s'écartaient de la norme de ses modèles. (Pamuk, Mon nom est Rouge, 2001, Folio : 674-675)

Trois conceptions différentes se font suite : la première repose sur l'approche singulière du style, à l'instar des théorisations relatives au style d'auteur, réduite ici à une dimension quasi symptomale : le style provient de «motivations profon- 
des », « cachées », bref, d'un écart défectueux face à une norme ${ }^{(5)}$. Dans cette optique, on n'a pas de style volontairement, c'est l'autre qui impute un style à un individu, avec du recul, le plus souvent. Les deux autres conceptions sont davantage en lien avec l'approche socio-culturelle : le style est ainsi défini comme caractéristique commune à un groupe plus ou moins large, allant du «style d'atelier » au style « national », voire au style « supranational» et transhistorique (ou « religieux $»{ }^{(6)}$ ) de la peinture islamique ${ }^{(7)}$ en opposition à « la » ${ }^{(8)}$ peinture européenne qui utilise la perspective ( « les choses n'y ét[ant] pas peintes à la mesure de leur importance au regard de Dieu, mais comme nous les percevons, et comme les peignent les Européens » (ibid. : 701-702)), en une sorte de simplification qui subsume les traits nationaux (à supposer que l'idée de style national, en lien avec le génie national des peuples, de leur langue et de leur culture, soit une idée encore pertinente de nos jours ${ }^{(9)}$ ). Il est intéressant de souligner que dans tous les cas, la valeur d'un style n'est pas une donnée intrinsèque, elle ne fait sens que contrastivement : dans le premier cas, il y faut le recul du temps : ainsi ce qui paraissait une « erreur » ou une « faiblesse » pour « les maîtres anciens » acquiert ses « lettres de noblesse ». Dans les autres cas, il faut le recul esthétique et/ou idéologique, la comparaison avec une autre manière de faire, qui, par contrecoup, assigne une singularité et une signification à ce qui paraissait jusqu'alors comme une pratique naturelle inintentionnelle ${ }^{(10)}$.

Anna Jaubert insistait, dans un précédent travail sur le style et ici-même, sur les tensions qui le traversent, entre un pôle singularisant (un style, comme ensemble de traits génériques, ou le style, comme forme singulière) et un pôle universalisant ( $d u$ style), sans qu'il y ait une contradiction entre le singulier pluralisable ( $u n$ style) et le singulier massif ( $d u$ style), ces deux acceptions s'articulant dans l'exercice du langage (Jaubert, $2005: 39-40)$. Oublier cette dialectique revient à alimenter les représentations dominantes du style, autour de la dissociation dualiste du fond et de la forme, qui a nourri les approches discontinues du style, les théorisations du style comme écart significatif par rapport à une norme, ou, en amont, les conceptions rhétoriques du style comme réservoir de manières de dire (c'est la théorie des registres, ou des styles sublime, moyen ou bas, telle du moins qu'elle a été présentée depuis le Moyen Age), réduites le plus souvent aux tropes. Ainsi qu'on l'a montré dans un article antérieur (Rabatel, 2007), la logique dua-

(5) $C f$. «Mon Oncle, lui ai-je répondu, disait qu'une erreur qui ne provient pas d'un manque de maîtrise, mais émane de l'intérieur de l'âme de l'artiste, cesse d'être une erreur, et devient un style. » (Pamuk, Mon nom est Rouge, 2001, Folio : 486).

(6) Il ne s'agit que d'une approximation sans fondement scientifique.

(7) En passant par un style d'Ecole, comme celui de l'Ecole de Hérat, évoqué dans la citation suivante : «Car s'il est permis à notre art d'être décoratif, notre foi interdit qu'il reproduise l'apparence des choses. Les peintures des anciens maîtres, y compris ceux de Hérat, étaient une forme de décor en marge du texte, d'enluminure, et personne n'y trouvait à redire, car il s'agissait d'exalter l'art du calligraphe. De plus, à combien de personnes en tout est-il donné de voir nos œuvres? N'empêche que, avec le recours aux méthodes venues d'Occident, notre peinture perd son rôle simplement ornemental, pour devenir franchement descriptive. Et c'est précisément ce que défend le Coran Vénérable. » (Pamuk, Mon nom est Rouge, 2001, Folio : 703-704).

(8) «La » peinture européenne d'une certaine époque, bien évidemment... Il est certain qu'avec ce genre d'extension, les caractéristiques stylistiques historiquement datées sont considérées comme non significatives, tandis que certaines caractéristiques esthétiques sont absolutisées : cf. supral'analyse de Genette.

(9) Ces représentations avaient cours aux XVIII et XIX ${ }^{\mathrm{e}}$ siècles. Cf. Huchette, 2001.

(10) Ces phénomènes de distance peuvent se combiner, comme en (4). 
liste n'est pas consubstantielle aux théorisations rhétoriques du style, mais elle a durablement accompagné les réductions qui ont jalonné l' histoire de la rhétorique (Amossy, 2006).

Nous ne reviendrons pas ici sur l'histoire de cette dérive dualiste, pas plus que sur celle qui a marqué les approches herméneutiques du style d'auteur. Nous préférons nous attarder sur un certain nombre de problèmes relatifs à la notion de style d'auteur - insuffisamment questionnée dans la formation initiale des enseignants -, avant de proposer une approche moniste du style et de l'exemplifier à partir de la problématique des figures et de la figuralité.

\subsection{En amont de la notion complexe de style d'auteur, la notion problématique d'auteur}

Parler de style d'auteur présuppose que la notion d'auteur soit bien établie et stable, ce qui est loin d'être avéré. Les travaux de Barthes et de Foucault montrent que l'auteur ${ }^{(11)}$ est une notion récente, et, pour tout dire, fortement mise en cause dans certains domaines scientifiques, techniques, juridiques, politiques, etc., mais aussi, de plus en plus, dans les domaines artistiques.

Barthes rappelle que la figure de l'auteur est très récente dans l'histoire, en lien avec d'une part l'invention de l'imprimerie, et d'autre part un certain nombre d'évolutions politiques, sociales et culturelles qui ont favorisé son émergence aux alentours de la Renaissance. En effet, jusqu'au moyen âge, dans les communautés d'érudits rassemblés autour des textes recopiés par les copistes, la notion d'auteur est proche de celle des sociétés orales : on distingue le scriptor du commentator du compilator, mais pas d'auctor au sens moderne du mot : ces rôles renvoient à un intellectuel collectif qui se fonde sur une pensée collective qui se transmet collectivement. C'est plus tard qu'émergent complémentairement les notions d'individu et d'auteur (Barthes, $1970: 184-185)^{(12)}$.

$\mathrm{Au} \mathrm{XX}^{\mathrm{e}}$ siècle, Foucault, notamment dans sa conférence «Qu'est-ce qu'un auteur? ", est un de ceux qui a puissamment contribué à la réflexion sur les évolutions de la figure de l'auteur, dans les arts comme dans les sciences. La figure de l'auteur, comme la fonction-auteur, change d'objectif, par rapport à 1'approche traditionnelle de l'auteur-démiurge, au point de ne peut plus pouvoir être appréhendée à partir de l'image d'un sujet originaire unique (Foucault, Dits et écrits 1 : 543, 838-839, Dits et écrits 2, 28-40). La fonction auteur est ainsi radicalement remise en question, compte tenu du dialogisme radical de la langue, de l'interdiscours qui structure tous les échanges, de la dimension collective des pratiques scientifiques et artistiques (davantage dans celles-là que dans celles-ci, certes), ce qui remet en cause le caractère idéologique de la notion d'auteur-fondateur, tant du point de vue des auteurs que des récepteurs, qui participent activement aux configurations et reconfigurations de la fonction auteur.

(11) A ce stade de généralité, on laisse de côté la distinction entre auteur et écrivain, telle qu'elle se trouve théorisée par exemple par Danon-Boileau, 1982.

(12) Mais aussi de sujet. Sur ce point, on lira avec profit la mise au point de Bernadet, 2001 : le sujet n'est pas le double de l'individu, c'est, ainsi que Meschonnic l'a bien vu, un mode de spécification de 1'individu : «Si le sujet présuppose toujours l'individu, il peut le transformer [...] Que le sujet s'inscrive naturellement dans l'histoire dans la mesure où il y est déjà impliqué par l'individu n'empêche pas qu'il transcende cette situation » (Bernadet, 2001 : 16). Dans le droit fil de ce raisonnement, l'auteur est lui aussi un des modes de spécification de l'individu et une aspects de sa subjectivation. Sur la naissance de l'écrivain, $c f$. notamment Viala, 1985 et Bourdieu, 1992. 
On pourrait en conclure que la notion d'auteur ne fait sens que dans le domaine littéraire (ou artistique, plus généralement). Certes, elle y résiste davantage que dans d'autres champs, mais cela doit être relativisé par le dialogisme foncier de la langue, la prégnance des phénomènes intertextuels, qui complexifient la notion et invitent à la circonscrire fortement dans le champ littéraire ${ }^{(13)}$. Les travaux de Genette sur l'intertextualité rappellent que la voix de l'auteur émerge constamment en écho de voix antérieures, tout comme ceux de Bakhtine sur la polyphonie soulignent l'intrication de voix auctoriales et actoriales (Rabatel 2006b, c). Dans ces conditions, isoler le propre de l'auteur relève de choix qui peuvent se comprendre, mais qui ne doivent pas faire oublier que cette logique conduit à laisser beaucoup de choses de côté, ainsi que l'écrit Genette, qui aime rapporter l'agacement d'Ingres, quand on voulait le réduire à une manière de peindre (« Monsieur, j’ai plusieurs pinceaux », apud Genette, $2006: 337)$.

La complexité de la notion d'auteur rend plus opaque la notion de style d'auteur, surtout si on la fait reposer sur une sélection de traits discontinus. Cette sélection fait problème par rapport à tout ce qui est laissé dans 1'ombre, considéré comme non significatif. Mais elle fait aussi problème par rapport au statut des traits jugés singuliers, car il leur arrive de renvoyer autant à un individu qu'à une époque ou à une école (Philippe 2005). Au fond, un bon test revient à pratiquer, à partir d'extrait peu connus, des lectures « à l'aveugle», et à rechercher leur auteur, sur la base d'indices linguistiques, stylistiques ou thématiques en faisant une totale abstraction de données biographiques : cette « désauctorialisation » de la lecture, au sens où celle-ci serait marquée par le déterminisme biographique (Genette, $2006: 47-48$ ), est un exercice salutaire qui montre que, parfois, les seuls traits stylistiques permettent davantage de donner une constellation de noms relevant d'une même esthétique, qu'ils ne suffisent à déterminer un nom - surtout si le jeu consiste à réfléchir sur des extraits d'auteurs de second rang comportant un certain nombre de traits que les pratiques scolaires n'attribuent qu'à tel grand écrivain...

On comprend que l'approche du style d'auteur soit doublement problématique - ce qui n'enlève rien au bien fondé de vouloir rendre compte de telle ou telle parole singulière. Mais cela passe par d'autres conceptions que le choix de marques discontinues assorties de considérations biographiques qui relèvent souvent du bricolage téléonomiste, articulées avec la stylistique des genres ${ }^{(14)}$. Mais il va de soi que l'approche génétique des textes est tout à fait en capacité d'échapper à ces réductionnismes, si elle s'intéresse à la totalité significative du matériau verbal et si elle rend compte des tensions singularisantes par lesquelles un écrivain manifeste un style original à travers le traitement spécifique de données stylistiques génériques.

\subsection{Pour une approche moniste du style}

Seule l'approche moniste est de nature à rendre compte de l'ensemble des tensions qui configurent la problématique du style. De quoi s'agit-il ? L'approche

(13) Dans le domaine artistique, la fonction auteur est de plus en plus bouleversée avec la multiplication des intervenants au cours et autour du processus créateur.

(14) La notion de stylistique des genres, héritière de Chklovski, Tynianov et Bakhtine (Combe, 2002 : 35-36), présente l'avantage d'une articulation entre la stylistique de la langue (Bally) et celle des auteurs (Spitzer), ainsi que le souligne Combe, 2002: 43-47. 
moniste de la langue et de la pensée repose sur le refus de saisir comme des entités autonomes la pensée et le langage, le vouloir dire et le dire ${ }^{(15)}$ :

(5) Il n'y a pas la pensée et le langage, chacun des deux ordres à l'examen se dédouble et envoie un rameau dans l'autre [...] Les opérations expressives se passent entre parole pensante et pensée parlante, et non pas, comme on le dit légèrement, entre pensée et langage. Ce n'est pas parce qu'ils sont parallèles que nous parlons, c'est parce que nous parlons qu'ils sont parallèles. (Merleau-Ponty, Signes, Gallimard $1960: 26)$

Sans entrer dans le développement des idées de Merleau-Ponty sur la relation entre le langage, la pensée et l'expérience sensible de l'être-au-monde (Rabatel, 2007), retenons en ici une conclusion pratique relative au refus de dissocier la forme et le fond :

(6) On a bien raison de condamner le formalisme, mais on oublie d'habitude que son tort n'est pas d'estimer trop la forme, mais de l'estimer si peu qu'il la détache du sens. En quoi il n'est pas différent d'une littérature du « sujet », qui, elle aussi, sépare le sens de l'œuvre de sa configuration. Le vrai contraire du formalisme est une bonne théorie du style, ou de la parole, qui les mette au-dessus de la « technique » ou de l' « instrument». La parole n'est pas un moyen au service d'une fin extérieure, elle a en elle-même sa règle d'emploi, sa morale, sa vue du monde, comme un geste quelquefois porte toute la vérité d'un homme. Cet usage vivant du langage, ignoré du formalisme aussi bien que de la littérature à « sujets », est la littérature même comme recherche et acquisition. Un langage, en effet, qui ne chercherait qu'à reproduire les choses mêmes, si importantes soient-elles, épuiserait son pouvoir d'enseignement dans des énoncés de fait. Un langage au contraire qui donne nos perspectives sur les choses et ménage en elles un relief inaugure une discussion qui ne finit pas avec lui et suscite lui-même la recherche (MerleauPonty, «Le langage indirect et les voix du silence », Signes [1960], 2001, Folio Essais : 124-125).

Le dualisme du style est souvent alimenté par l'analyse rhétorique des figures ${ }^{(16)}$, appréhendées à partir des notions d'écart par rapport à une norme, la valeur y étant calculée par rapport aux notions précédentes et non en fonction de l'unité forme-sens, comme dans l'approche moniste de Meschonnic. Les théories substitutives présentent le défaut d'une approche décontextualisée et paradigmatisante (qui alimente les dictionnaires et autres glossaires de termes de rhétorique), réduite aux tropes ${ }^{(17)}$, survalorisant quelques figures d'élocution (métaphore, méto-

(15) Meschonnic en donne une définition similaire, quoique plus (trop ?) globale, en y incluant l'indissociabilité du vivre et du dire : «Monisme (matérialisme) : Homogénéité et indissociabilité de la pensée et du langage, de la langue et de la parole, de la parole et de la graphie, du signifiant et du signifié, du langage et du métalangage, du vivre et du dire. S'oppose à dualisme, hétérogénéité du signifiant et du signifié, de l'expression et du contenu, de la langue et du style, du sens et du style... Le monisme matérialiste se définit la condition de production des formes-sens. » (Meschonnic, 1970:177).

(16) «Une figure est une forme discursive marquée, libre et mesurable qui renforce le rendement des énoncés », selon la définition de Bonhomme, 1998: 7.

(17) $C f$. l'opposition entre «tropes en un seul mot» $v s$ " tropes diffus ». Cette opposition est contestée par tous ceux qui refusent d'établir une différence entre trope en un seul mot et trope diffus, à l'instar de ceux qui prônent une approche pragmatique des figures. C'est par exemple le cas de Perrin pour qui il n'existe pas de différence entre trope en un seul mot et trope diffus : « les tropes ne sont jamais autre chose que ce que les Anciens ont défini comme des figures de pensée. L'erreur fondamentale des rhétoriciens n'était pas de rapprocher l'ironie de la métaphore et d'en faire une figure de rhétorique, mais bien d'opposer les 
nymie, etc.), au détriment des figures de pensée et d'invention, ce qui in fine alimente les approches dualiste de la langue et du style, puisque les figures n'affecteraient pas le fond de la pensée (laissant intacts les contenus propositionnels des énoncés) et se limiteraient à des tours de phrase qui ne visent que la «grâce », laissant dans limbes les intentions pragma-énonciatives des locuteurs. Une telle conception a la vie dure, comme le montre l'extrait suivant, dont la dimension humoristique et le parler oral contemporain s'accommodent néanmoins d'une conception du style très ancienne :

(7) Et par exemple comment on appelle les grands couturiers, ou ceux qui inventent les vêtements? On les appelle des stylistes. C'est-à-dire que vouloir être beau niveau vêtements, c'est faire attention au style, Ndeyé tais-toi. En gros, le style c'est tout ce qui n'est pas strictement utile. Eh bien pour le langage c'est pareil. Je peux dire quelque chose en me contentant de l'information que je veux transmettre, par exemple je suis né en France. Mais je peux dire la même chose en ajoutant du style, par exemple je suis né au pays des fromages, ou au pays des droits de l'homme. Là, je fais du style, du mauvais style mais du style. Et pour çaj'utilise un procédé, et ce procédé il a un nom, Ndeyé qu'est-ce que j'ai dit? Par exemple, quand je vais à la patinoire, je peux me contenter de tourner sur la glace, comme on fait tous quand on est pas champion. Mais les champions eux ils font quoi ? Ils font des figures, des triples-flip et tout ça, Ndeyé c'est la dernière fois. Dire «pays des droits de l'homme » à la place de France, cela s'appelle faire une figure de style, et des figures de style il y en a plein ? Celle-là c'est la périphrase. (Bégaudeau, Entre les murs, Verticales, 2006 : 198)

À rebours, 1'approche moniste des figures pense le fait figural non seulement dans ses continuités avec les usages ordinaires du langage, mais encore en rejet absolu d'une approche ornementale réservée aux «champions » de tous ordres. L'écart, s'il ne correspond pas à une manière oblique qui se substituerait à une manière directe de s'exprimer (représentation qui certes n'est jamais formulée si directement par les linguistes au fait du caractère idéal factice de cette représentation, mais qui a néanmoins pratiquement cours, comme le montre l'exemple précédent, qui n'a rien de caricatural), renvoie plutôt « à une actualisation inattendue [d'un point de vue sur tel objet de discours] au regard d'une signification intersubjectivement stable, et dans l'appropriation toute personnelle de cette signification, qui la rend plus ou moins étrange, selon que la figure est plus ou moins conventionnelle» (Détrie, 2000 : 9). La saisie de l'écart opère ici dans le cadre d'une sémantique référentielle dans laquelle « les mots effectuent des découpages non du monde réel, mais du monde vécu par les sujets parlants, les mots reflétant, véhiculant avec eux des expériences multiples du sensible ${ }^{(18)}$, expériences anthropologiques, manipulatives, ou culturelles » (ibid.). Dans ce cadre d'analyse, non seulement les figures (de rhétorique) font l'objet du même traitement énonciatif

tropes dans leur ensemble aux figures de pensée » (Perrin, 1996:15). Il y a néanmoins un intérêt au maintien de l'opposition, au plan descriptif : les tropes en un seul mot sont des tropes dont le foyer ne s'étend que sur un segment de la phrase et doublent l'incohérence avec le co(n)texte d'une incohérence interne. Dans les tropes diffus, c'est tout un énoncé qui reçoit une interprétation figurée sur la base d'un conflit avec le cotexte. Bref, les tropes diffus ne sont pas incohérents d'un point de vue interne, $c f$. l'allégorie ou les métaphores filées (Prandi, 2000). Une telle description n'est cependant pertinente que si l'on relativise la notion de déviance : car un énoncé linguistiquement déviant ne l'est pas nécessairement sur le plan discursif (Nyckees, 2000:119).

(18) Cf. les gestalts expérientielles chez Lakoff et Johnson, 1985. 
que n'importe quel autre mode de donation des référents, mais encore sont pensées dans le cadre d'une dialectique par laquelle le locuteur innove tout en jouant avec les règles d'appariement (ce que Bonhomme 2005 : 58-67 appelle des mécanismes de saillance) ainsi qu'avec des manières de voir plus ou moins conventionnelles.

L'analyse moniste des figures rejette la thèse substitutive de l'écart, même affaibli, ainsi que la notion de rupture d'isotopie ${ }^{(19)}$; elle interprète l'écart comme tension intradiscursive, entre des points de vue en confrontation ${ }^{(20)}$. La dialectique de l'innovation et de la convention, de la liberté et des règles (morpho-syntactico-sémantiques) est au cœur du processus de création figurale - « compromis de singularité (qui la rend remarquable) et de régularité (qui la rend mesurable sur la chaîne des énoncés) », selon Bonhomme 2005 : 70 - sans se distinguer radicalement du fonctionnement ordinaire du langage.

Autrement dit, les locuteurs s'approprient les trésors de la langue, des genres, des esthétiques et exercent leur liberté de locuteur dans le cadre du jeu avec des contraintes variées, à l'instar de la dialectique primat/primauté chez Comte-Sponville, 1988 : 302-305 : primat des données situationnelles, génériques structurantes (donc, primat du social, sous ses formes culturalisées), à l'intérieur desquelles s'exerce ensuite la primauté du vouloir dire du sujet/locuteur. Cette question, décisive, mérite d'être précisée. Dans ses efforts pour élaborer une philosophie matérialiste de 1'existence qui rende compte des aspirations humaines à se libérer en desserrant les contraintes qui l'entravent, tout en réservant aux mécanismes structurants du vivant leur place éminente, Comte-Sponville insiste sur cette dialectique du primat, qui accorde la première place aux mécanismes qui nous font être, et des primautés, par lesquelles l'homme refuse d'être le simple jouet des influences. Pour autant, il ne s'imagine pas au-dessus d'elles, en sorte que ses initiatives s'exercent dans le jeu complexe des déterminations et des contraintes ${ }^{(21)}$. Ainsi, l'homme matérialiste, sans nier le poids des déterminismes (physiologiques ou sociologiques), refuse de penser que ceux-ci ont le dernier mot, sans toutefois se berner d'illusions sur les limites de son pouvoir - mais non de son vouloir:

(8) Ce que j'ai appelé la dialectique du primat et de la primauté (par quoi le matérialisme se pense lui-même comme ascension) introduit un renversement, sinon des valeurs elles-mêmes (encore que cela puisse se produire), du moins de leur statut, et permet de les penser, sinon de toujours les vivre, de manière non religieuse. Le matérialisme est une entreprise de démystification: il s'agit de penser l'illusion en vérité. [...] Du moins commençons-nous à voir plus clairement l'ascension qu'il s'agit de penser : du primat (de la matière, de la nature, de l'économie, de la force, de l'inconscient, du monde...) à la primauté (de la pensée, de la culture, du sens...). Entre les deux : le mouvement ascendant du désir, qui nous fait passer de l'un (primat du primat : tout part du corps) à la primauté (primauté de la primauté : rien ne vaut que l'esprit). [...]

(19) La notion de rupture d'isotopie (Dubois et alii, 1970) ne rend pas compte de la globalité du fait figural, puisque nombre de figures, telles l'amplification, 1'hyperbole, etc., ne reposent pas sur une rupture d'isotopie, mais sur des changements de points de vue, à l'intérieur d'une isotopie constante.

(20) Notion qu'il convient d'articuler aux phénomènes de saillance discursive de Bonhomme.

(21) Cette manière de voir n'est pas sans relation avec les évolutions de la sociologie, qui, sans nier l'importance des facteurs (déterminations), mettent en avant le rôle des acteurs, dont les trajectoires influent sur les déterminations. 
Ce désir est un effort (conatus...), une tension, un acte. On ne passe du primat à la primauté qu'à la condition de le vouloir. Qu'un instant l'effort se relâche, que le désir se fatigue ou se lasse... on n'a plus qu'un matérialisme vulgaire, plat, avachi, un matérialisme qui redescend - et qui ne saurait par conséquent être philosophique.

(Comte-Sponville $1988: 302-304)$

Cette analyse offre un cadre très éclairant pour penser les tensions singularisantes du travail personnel des locuteurs sur le style (primauté de l'auteur et du style d'auteur) qui opèrent sur le fond structurant des choses à dire, de telle façon, dans telle situation (primat des genres). Plus exactement, ce matérialisme philosophique est à même de penser la dialectique entre le rôle préfigurant et configurant des primats (primat des formes, des thèmes, des situations, des genres) et le rôle reconfigurant des primautés (affirmation/construction du soi), par la manière spécifique que les individus ont de «s'approprier les héritages langagiers » et d'exploiter le réservoir des formes à leur disposition, comme on l'a entrevu supra à propos du mécanisme de création figurale.

Cette dialectique dynamique pense la notion de style d'auteur comme volonté, désir, ascension, n'existant que sur le fond du primat des genres, tandis que la primauté de l'aspiration intellectuelle et sensible à la singularité (fût-ce dans la manière de revendiquer un air de famille singulier), s'exprime à travers une petite musique faite d'appartenances et de singularités. Inversement, l'absence de cette dynamique ascensionnelle (de son désir ou de sa nécessité) explique la permanence des formes et des genres ${ }^{(22)}$, sans innovation (primat des formes sans primauté des acteurs), voire toutes les manifestations d'académisme et de dualisme qu'évoquait Merleau-Ponty (primat de la «forme » sans primauté du « fond»: artificialismes ; primat du «fond » sans primauté de la «forme» : vérismes, etc.). De tels déséquilibres peuvent jouer dans tous les sens, comme lorsqu'un individu s'affiche comme valeur absolue en déni ou ignorance des autres (primauté de «sa majesté le moi » sans primat de l'environnement socio-culturel), produisant des œuvres narcissiques ou faussement originales, etc.

Partant de là, le monisme permet de traiter de façon globale « le tout du style », selon une approche continuiste du phénomène linguistique de l'actualisation ${ }^{(23)}$,

- en ne limitant pas le style à des figures ${ }^{(24)}$ ou à des énoncés décontextualisés, mais en visant le discours dans sa totalité ;

- en ne se bornant pas aux approches discontinues du style, mais s'intéressant au tout de la parole comme ensemble stylistiquement significatif;

- en ne limitant pas l'analyse à l'elocutio, mais en y intégrant la dispositio et l'inventio (voire l'actio et la memoria, si l'on s'intéresse aux mises en voix/mise en scène des textes);

- en rendant compte des dimensions singulières et collectives du style ;

(22) Cette dynamique (ou son absence) concerne les genres littéraires comme les genres premiers.

(23) Voir ici même l'article d'A. Jaubert.

(24) Voire, réductionnisme au carré, en réduisant les figures aux seuls tropes, et en ne privilégiant dans ce cadre théorique que certains tropes, dont la métaphore, figure «littéraire » par excellence. $C f$. la parution d'une prochaine publication de Langue française, consacrée à 1'analyse pragma-énonciative des figures et aux figures d'énonciateurs, à paraître en 2008, avec des articles de C. Détrie, A. Jaubert, L. Gaudin, M. Monte, A. Rabatel, G. Salvan et B. Verine. 
- en traitant du style sous son versant expressif comme sous sa dimension communicationnelle ${ }^{(25)}$;

- en rendant compte du style comme recherche esthétique ou comme recherche de la fonctionnalité maximale (Rastier, 1994 : 274).

Ainsi le style est sans doute le lieu où se manifeste le mieux la dynamique de construction/spécification de soi à travers le retravail des formes sociales et culturelles par lesquelles les individus expriment leurs rapports entre eux, leur rapport au monde et leur rapport au langage. Dynamique d'affirmation de soi qui joue constamment sur des tensions entre reproduction et innovation, et qui opère au plan des grandes unités (textes, discours ${ }^{(26)}$ ) organisées selon des normes, des conventions fonctionnelles et esthétiques qui structurent les interactions de la vie quotidienne (genres premiers) comme celles qui y échappent et sont davantage soumises à un processus d'esthétisation autotélique (genres seconds, $c f$. Bakhtine, 1978).

Ce processus d'affirmation de soi, souvent érigé en valeur suprême dans nos sociétés occidentales, est ici appréhendé dans une dialectique par laquelle les individus, pour se faire reconnaître et désirer dans leur identité, leur valeur, imitent les valeurs et comportements (notamment langagiers) des autres, d'abord les parents, puis l'entourage, afin de se faire reconnaître par les autres, ce qui leur donne la force de s'autonomiser et de s'affirmer en tant que personne (Flahault 2006 : 78s, 158 s). Ainsi, la question du style est tout sauf une question purement techniciste : elle est socialisée de part en part, sur les plans psychologique, social, culturel. Autant dire que la dimension technique ne peut faire sens que d'un point de vue anthropologique qui prend en compte la globalité du processus de construction de soi, ce qui invite à préciser la part respective du style, de l'idiolecte et de l'ethos dans ce processus.

\section{Style, idiolecte et ethos}

$\mathrm{Si}$, avec la problématique du style, la dialectique du singulier et du social joue au plan des structures transphrastiques que sont les genres, est-ce à dire que les processus de singularisation idiolectale ou éthique opéreraient en déport du social, comme semble l'indiquer l'étymologie d'idiolecte ? Il n'en est rien, même si les données sociales contraignent autrement les processus de spécification des individus, en étant ressenties comme moins directement structurantes que les genres ou les sociolectes.

\subsection{Style et idiolecte}

Selon Rastier, 1994 : 276, il n'y a pas lieu d'opposer idiolecte et style, linguistique et stylistique, mais au contraire de les articuler :

(25) Meschonnic pose que la problématique de la valeur, à travers les formes-sens (Meschonnic, 1970 : 176), est «à son degré plein au niveau de la littérarité » (ibid. :175), ce qui ne veut pas dire que la valeur n'existe que dans les textes littéraires. Il n'en reste pas moins vrai que sa réflexion est fortement empreinte des débats des années 70 du siècle passé autour de la littérarité.

(26) Les grandes unités intégrant les plus petites, à l'instar des figures de pensée ou des tropes, par exemple. 


\begin{tabular}{|c|llll|}
\hline (9) & Entours & $\begin{array}{l}\text { pratique } \\
\text { linguistique }\end{array}$ & $\begin{array}{l}\text { pratique } \\
\text { sociale }\end{array}$ & usage (déterminé) \\
\cline { 2 - 5 } & Instances & langue & discours & parole \\
Formations & dialectes & sociolectes & idiolectes \\
Formes & textualité & genre & styles \\
Degrés de & système & normes & normes \\
Systématicité & fonctionnel & sociales & individuelles \\
Disciplines & linguistique & poétique, linguistique \\
Actuelles & restreinte & des discours & stylistique \\
\cline { 3 - 5 } & & & \\
\hline
\end{tabular}

Ce tableau présente une tentative d'articulation des notions de style et d'idiolecte et aussi une tentative d'intégration de la stylistique à la linguistique, à travers la systématisation des relations hiérarchiques entre le système fonctionnel de la langue, les normes sociales et les usages personnels. Si les idiolectes littéraires sont les plus systématisés, du fait de la prégnance des normes littéraires, « rien ne permet, sauf préjugé esthétique souvent légitime, d'écarter les autres formations idiolectales ${ }^{(27)}$. En d'autres termes, si l'on convient de nommer styles toutes les formations idiolectales, les styles littéraires ne comptent que pour une part des styles linguistiques » (Rastier, $1994: 276$, repris sous une forme approchante in Rastier, 2001 : 179). Cette représentation, qui rompt avec les conceptions dogmatiques de la langue et le mysticisme de l'individu créateur (Rastier, 1994 : 279), semble a priori satisfaisante pour la notion de style, parce que l'idiolecte s'y présente (paré d'un ethos de scientificité), comme le concept idoine pour mettre de l'ordre dans la confusion du style... Ce qui présuppose que l'idiolecte soit un concept clair : or, comme le remarquent les éditeurs d'une récente publication stimulante consacrée à l'idiolecte, la stabilité définitionnelle de la notion ne l'a pas prémunie contre le flou (Détrie et Neveu, $2005: 7$ )...

En dépit des propositions de Rastier, 1994 et 2001, on mesure la difficulté de fonder en langue la notion protéiforme de style grâce à celle d'idiolecte, d'une part parce que l'idiolecte ne se laisse pas circonscrire si aisément que cela en raison de l'inconsistance de la notion de sème afférent, d'autre part parce que l'idée d'une approche scientifique de la parole singulière, à supposer qu'on puisse la mener à terme, ne représente qu'un des aspects de la notion de style. Même si nous n'avons pas la place de traiter au fond de l'idiolecte (nous renvoyons à Détrie et Neveu 2005), au moins peut-on esquisser une approche des relations entre idiolecte et style qui ne se réduise pas à un pur recouvrement, comme le proposait Rastier. Outre que le style ne se réduit pas à la dimension langagière, à la différence de

(27) Au demeurant fort peu étudiées, non seulement parce qu'une linguistique de la parole (au sens saussurien) continue d'inquiéter, mais parce qu'une linguistique différentielle, homologue de la psychologie différentielle, reste inconcevable pour les linguistiques universelles qui tiennent encore le devant de la scène académique. Le statut épistémologique de la linguistique se trouve ici mis en jeu : une discipline formelle ne peut naturellement pas rendre compte du particulier, et interprète en son sens le plus fort la maxime aristotélicienne qu'il n'est de science que du général. En revanche, une discipline descriptive trouve son aboutissement dans la description du particulier. (Note de Rastier 1994: 276). 
1'idiolecte, il est d'application plus large que ce dernier, au plan langagier : on ne parle pas d'idiolecte romantique ou d'idiolecte sublime, on change plus facilement de style que d'idiolecte, etc.

L'idiolecte, comme le style, mais davantage que lui, rend compte de la subjectivité des individus à travers leurs identités langagières. Il aide à penser les processus de tension singularisante ${ }^{(28)}$ configurés par la dimension sociale du matériau langagier et par le jeu interactionnel, afin de rendre compte de la co-construction interactionnelle des identités et des singularités langagières (Détrie, 2005, Rabatel, 2005b). L'idiolecte, mieux que le style, permet de penser le singulier dans le langage. Il peut même alimenter des styles, comme il alimente des sociolectes, ainsi que l'avait entrevu Barthes 1969, 1984, si l'on en tient, comme c'est notre cas, pour une conception dérivationnelle et sociale du style (Détrie et Neveu $2005: 10$ ).

Néanmoins, l'idiolecte est mal nommé, n'étant pas un «lecte » comme les autres (l'idée d'une langue singulière étant une aporie que ne se sont pas fait faute de souligner les adversaires de la notion, au point que Gadet, 2003 : 125 propose de définir l'idiolecte comme « une idéalisation de la façon de parler spécifique du locuteur »). La dimension pragmatique de l'idiolecte explique la variabilité des motivations de la re-présentation, ainsi que la variabilité de ses formes (fidèle ou fausse, complète ou pointilliste, de bonne ou de mauvaise foi). Elle explique aussi le fait que n'importe quel élément linguistique soumis à variation puisse faire l'objet d'un traitement idiolectal ( $c f$. le tableau sur le matériau variationnel en français chez Gadet, 2003 : 44). Autrement dit, l'idiolecte ne fait sens que dans l'interaction : on choisit toujours de re-présenter l'autre, ou de se re-présenter en fonction de ses propres intérêts et de la nature de la relation que l'on noue avec autrui, ou en fonction de la façon plus ou moins singularisante ou non par laquelle l'on envisage de faire référence à tel objet de discours. C'est pourquoi il est somme toute assez courant d'enfermer l'autre dans une re-présentation qui n'est certes pas tout à fait fausse, mais qui devient forcée d'être réitérée à tout propos, voire hors de propos, comme si l'autre (ou l'autre en nous) était toujours tel que nous le disons, tel que nous le voyons ou voulons que les autres le voient. Il y a toujours de la violence dans cette re-présentation, tant du point de vue de l'idiolecté ${ }^{(29)}$, qui risque de ne pas se reconnaître (ou d'y perdre son crédit s'il donne de luimême une image trop calculée), que pour les destinataires réels ou secondaires de cette re-présentation qui, s'ils ne partagent pas l'image de l'autre qui leur est proposée, voient en elle une atteinte à la vérité ou à leur désir... En définitive, même si la construction d'une parole singulière par le langage est en tension entre soi et autrui, et n'ignore pas le social, elle opère, comme son nom l'indique, en déport du social, dans un processus de dimension interactionnelle / coénonciative dont l'empan est restreint à la parole vive, et qui traite du social à travers la dialectique de l'identité, de la mêmeté et de l'altérité (Détrie 2005, Rabatel 2005b).

\subsection{Style et ethos}

Quelle place pour l'ethos, dans cette configuration ? La thèse qu'on voudrait défendre est que l'ethos rebrasse les cartes du social et du singulier en les subor-

(28) C'est pourquoi Détrie 2005 parle de dynamique idiolectalisante, entre singularisation et réitération.

(29) Nous distinguons deux rôles fonctionnels : l'idiolecté fait l'objet d'un traitement idiolectal et l'idiolectant est à l'origine de ce traitement (Rabatel 2005b). 
donnant à une visée argumentative. Si l'on se réfère aux origines de la notion, l'ethos vise la manifestation d'un dire vrai dans une relation où locuteur et destinataires du propos sont physiquement en face à face, pour délibérer ou juger. Même si l'extension de la notion à la présence du sujet dans un texte écrit est légitime, elle ne doit pas faire oublier la dimension interactionnelle première de la notion, et son horizon argumentatif. La manifestation de la vérité du sujet ne vaut que par rapport à un point de vue à faire valoir, ainsi qu'il apparaît dès les premières théorisations de l'ethos dans l'antiquité grecque, puis chez les Romains. Certes, on pourrait discuter la pertinence du retour à Aristote ou à Cicéron, mais il nous semble que les approches contemporaines de l'ethos (théories énonciatives, interactionnelles, analyse du discours $c f$. Amossy, 2006), mettent souvent en avant l'expressivité (du locuteur en tant que tel, ou du locuteur / interactant) sans articuler suffisamment celle-ci avec sa dimension argumentative (directe ou indirecte). D'où notre insistance sur la spécificité des tensions argumentatives et expressives d'une parole qui, pour persuader et convaincre, joue sur les claviers complémentaires des valeurs expressives et logiques, valeurs, qui sont toutes deux traversées par des variables individuelles et sociales.

L'ethos aristotélicien a une double dimension : au sens moral, basé sur l'epieíkeia, il englobe les vertus (honnêteté, bienséance, équité) du sujet ; en un sens dit «neutre» (parce moins en prise sur le sujet singulier), il renvoie à des notions évoquées à travers les expressions habitudes, mours, coutumes ou caractères, bref, des comportements (héxis) propres à un groupe ou une fonction, que l'auditoire s'attend à reconnaître dans telle circonstance. Ces deux conceptions ne s'excluent pas, au contraire, ce sont deux « faces nécessaires de toute activité argumentative » (Eggs, $1999: 32)$.

(10) Les orateurs inspirent confiance pour trois raisons : ce sont, en effet, les raisons qui déterminent notre conviction en dehors des démonstrations (apódeixis) : (a) la prudence/la sagesse pratique (phrónesis), (b) la vertu (areté) et (c) la bienveillance (eúnoia). (Rhétorique, II, 1378a 6), apud Eggs, 1999 : 35).

Autrement dit, l'ethos chez Aristote repose sur « un mélange de psychologie et de stratégie sociale et discursive » : cette double dimension, individuelle et sociale, n'a cessé d'alimenter les approches contemporaines de l'ethos, en tant que « mélange complexe de la personne réelle, de l'image qu'elle offre au public, de sa réputation, de son charisme, du rôle qu'elle occupe au sein de l'institution et de la palette infinie des mises en scène qu'elle peut produire en jouant avec les règles et les normes sousjacentes à toute situation rhétorique » (Danblon, 2006 : 132). On trouve des échos de ce «mélange » chez Amossy, 1999, avec la notion d'ethos préalable, chez Maingueneau, 1999 avec celle d'ethos prédiscursif, chez Meyer, 2004 avec celles d'ethos projectif et d'ethos effectif, qui renvoient aux différences entre les images construites par l'orateur et l'auditoire. Cette dialectique de l'individuel et du social se retrouve également dans l'approche de la notion d'incorporation chez Maingueneau, 1999 : 79-80 : en effet, si la notion renvoie à la manière dont l'énonciation du texte confère une corporalité au garant, elle renvoie aussi à la mise en œuvre de schèmes corporels qui font sens pour le coénonciateur, et, en troisième lieu, elle renvoie de surcroît à la construction d'un corps social formé de la communauté de ceux qui adhèrent à tel discours et à telle manière de le vivre.

Danblon 2006 : 21 résume bien la complexité de la notion à travers la dimension indiciaire de l'ethos, aux yeux de l'auditoire, certaines données personnelles ne faisant sens que pour un auditoire ou une société donnée, dans des circonstances 
données. Ces indices de caractère et d'émotion sont le gage des qualités de l'orateur et de sa sincérité. Ils sont changeants, selon les situations et les auditoires, alors qu'il n'en va pas de même pour les qualités idiosyncrasiques des individus. Mais tout extérieurs et sociaux qu'ils soient, ils n'en manifestent pas moins leur aptitude à s'incarner dans un individu et à coller avec lui, dans la situation où ils sont convoqués, s'ils sont bien mis en scène : alors, ils confèrent une valeur surajoutée (dans l'ordre de l'argumentation et de la persuasion) à celui qui en use efficacement ${ }^{(30)}$. Ces marques ne relèvent pas seulement de l'arrangement du discours, elles reposent aussi sur la voix et le corps. Ainsi en juge l'auteur de la Rhétorique à Herrennius, qui, à peu près sensiblement à la même époque que Cicéron écrivait son De inventione, consacrait l'essentiel du livre III à l'actio, et au travail conjoint de la voix et du corps, avant de traiter des styles et des figures de mots et de pensée dans le livre IV. Le texte mériterait d'être longuement cité - mais nous devons y renoncer, faute de place -, tant il fait ressortir l'approche globale des marques, traitant d'abord de la puissance et de la résistance de la voix avant d'aborder les questions relatives à sa souplesse, autrement dit à la mélodie et à la prosodie (Rhétorique à Herrennius, 1989 : 107-113). La minutie avec laquelle la prosodie, la mélodie, les gestes et les postures sont articulés avec les parties du discours et les modes souligne la dimension radicalement argumentative de ces outils, allant au-delà de leur dimension idiosyncrasique qui en forme le substrat.

Cicéron est certainement un de ceux qui ont le plus insisté sur cette dimension sociale incarnée de l'ethos, dans le travail de l'orateur idéal en tant que personnage public (orateur, avocat, homme politique). Cette dimension sociale, très forte chez les Romains, ne doit toutefois pas être interprétée comme un rôle que jouerait facticement l'orateur : c'est une des thèses fondamentale du De oratore que d'insister (à travers le discours de Crassus) sur le fait que les moyens de la rhétorique, sans être absolument condamnables, comme le pensent nombre de philosophes antiques, doivent prolonger la science, la pensée, chez l'orateur idéal. Ainsi, Cicéron écrit-il, à propos du conciliare :

(11) Ce qui nous concilie la bienveillance, c'est la dignité de notre caractère, ce sont nos actions louables, la considération qu'inspire notre vie : toutes choses qu'il est plus facile d'embellir lorsqu'elles existent, que de feindre quand elles n'existent pas. D'autres qualités ajoutent chez l'orateur à l'effet produit : la douceur de la voix, l'air du visage, l'aménité de la parole, l'impression que, s'il se livre à une attaque un peu vive, c'est à regret et par devoir » (De l'orateur, Livre II, XLIII, T. 2 : 79) ${ }^{(31)}$.

Et cette union de ce qui est dit et montré (comme de l'ethos et du pathos) est également développée à propos du movere :

(12) Jamais je n'ai essayé d'inspirer aux juges la douleur, la piété, l'envie ou la haine, que moi-même, le moment venu de les ébranler, je n'aie vivement ressenti pour mon compte les émotions que je voulais faire passer dans leur âme» (ibid. : 83).

(30) Ils relèvent des origines magiques et/ou archaïques dans lesquelles certains manières de parler ( $c f$. les formules magiques) et certains attributs externes (toge, skeptron, blouse, costume trois pièces, uniforme...) confèrent une autorité institutionnelle à la parole de celui qui parle, puisque c'est une autorité qui parle par sa voix. Ainsi de l'apparition de de Gaulle en uniforme, à la télévision, après le putsch des généraux à Alger, alors qu'en principe le général de Gaulle portait l'habit civil (Danblon, $2006: 21$ ).

(31) $C f$. encore Livre 1, XXIV, T.1: 110, XXXII, T. $1: 146$. C'est également la thèse de l'Orateur, que Cicéron écrit en -46 . 
Quintilien tient un discours similaire, à travers la mise en avant du caractère moral de l'orateur (« vir bonus dicendi peritus, optima sentiens, optimeque dicens »), garant de l'ethos d'honnêteté et d'intégrité, valeurs associées au modèle de l'éloquence latine. La dimension sociale des vertus représentées par l'orateur explique que l'ethos (comme le pathos, d'ailleurs) puisse être analysé comme des conventions, qui ne se réduisent pas à des stratégies manipulatives. C'est ce que souligne l'articulation que Quintilien opère entre ethos et urbanitas, c'est-à-dire un « mélange » de « bonté », « calme et douceur », de " gentillesse et humanité », d' " agrément et charme pour les auditeurs, et la perfection consiste à l'exprimer de telle sorte que tout donne l'impression de dériver de la nature des faits et des personnes, qu'y éclate à travers sa parole, et, dans une certaine mesure, s'y reconnaisse le caractère de l'orateur » (Quintilien, Institution oratoire Livre VI, 2, 13, $1977: 26 ; c f .61-62)$.

Il est habituel de dire que l'ethos, chez les Romains, est moins directement appréhendé sur le terrain proprement linguistique que sur celui du comportement et des mœurs. Si l'accent est ainsi mis sur ces dimensions extralinguistiques, en tant que technique que doit maîtriser l'orateur idéal, il ne faut pas sous-estimer le lien entre moyens extralinguistiques et linguistiques : ils vont de pair chez les Romains, et le fait qu'ils n'en parlent pas autant que les Grecs signifie moins un désintérêt qu'un accord profond, au plan théorique ${ }^{(32)}$.

L'ethos, c'est l'incarnation, dans le corps parlant, des valeurs sociales propres à persuader et à convaincre. Et c'est au plan social que se joue son efficacité, comme les Romains l'ont magistralement théorisé. C'est pourquoi l'ethos du propagandiste ou l'ethos prophétique ${ }^{(33)}$ ne fonctionnent que si les locuteurs incarnent les valeurs propres à ces ethe par leur charisme, d'une part et si, d'autre part, ces valeurs font sens pour leurs auditoires.

\section{Conclusion}

Si tant est qu'on puisse isoler une spécificité du style, par rapport aux notions affines de l'idiolecte et de l'ethos, il semble que ce soit dans l'importance consti-

(32) On peut néanmoins relativiser ce qui précède, si l'on distingue entre la visée idéale et la réalité des pratiques des orateurs, sous Quintilien, c'est-à-dire à une époque caractérisée par la décadence de l'éloquence depuis Cicéron (Institution oratoire, T.1 : XLVI-LV, LXXIV, et, surtout, T.4, Livre XI, 3). Dans ce cas, l'ethos vise moins un idéal qu'il ne fait écho aux pratiques réelles des individus, plus sujettes aux distorsions entre l'être et le paraître...

(33) L'ethos pamphlétaire ( $c f$. Angenot, 1982), se construit en position externe. Le pamphlétaire est un marginal (à l'instar d'Antigone), « libre, et donc authentique puisqu'il n'a rien à obtenir de l'institution. ". L'ethos du pamphlétaire ne s'appuie pas au départ sur une dimension religieuse, mais sa position atopique, et la construction de la vérité en opposition au groupe, renvoie à un ethos du solitaire lucide qui ressemble à celui d'un prophète... laïc (cf. Danblon, 2006 : 55). A l'opposé, «l'ethos du propagandiste - lorsque la propagande est officielle - se construit sur le modèle du prophète ou du devin du discours magique. Le discours n'admettant aucune critique, l'ethos du propagandiste est totalement intégré à l'institution au point de 1'incarner : L'Etat, c'est lui. »(Danblon, 2006 : 173-174). Le pamphlétaire comme le propagandiste, utilisent des arguments éthiques qui échappent à la discussion rationnelle. La prétention à la détention de la vérité les fait échapper à la charge de la preuve, ils jouent sur les émotions des évidences factuelles. Dans ces situations, il est impossible de discuter, réfuter, argumenter : on adhère, ou on est rejeté comme indigne de la nouvelle vérité. 
tutive structurante des notions conjointes de situation, d'auditoire, de sujet, fondamentales pour l'analyse des genres, et du style de tel ou tel genre : avoir un style, du style, c'est toujours dans une dialectique où chacun use de patterns, de modèles, de cadres, thèmes et situe son originalité dans la façon de rejouer ces derniers, dans une dialectique d'imitation ou d'opposition à tel genre ou à tel autre.

Là réside la différence fondamentale d'avec l'idiolecte. Certes, il n'est pas possible de faire abstraction des données situationnelles pour rendre compte de l'idiolecte ou de l'ethos, mais leur portée y est moindre, leurs données moins routinisées et moins objectivées dans des genres premiers, et, de ce fait, moins directement « lisibles » et contraignantes, dans nos sociétés occidentales, du moins, où les comportements, tout en étant socio-culturellement marqués, sont malgré tout ressentis par les individus comme déterminant moins ouvertement les interactions.

Avoir un style, du style ne se limite pas davantage à la visée argumentative caractéristique de l'ethos. Là encore, il n'est évidemment pas question de dire que le style ne se pose pas la question de l'ethos. Certes, le style d'un article scientifique obéit à des normes destinées à optimiser les dimensions informative et argumentative de la démonstration, et en ce sens, le style d'un article scientifique croise la problématique de l'ethos - mais cet ethos est proche de la phrónesis, bien plus que de l'areté ${ }^{(34)}$, reposant plus sur des règles de vraisemblables portant sur l'objet du discours que sur le sujet discoureur, qui doit s'effacer.

Bref, si l'idiolecte, c'est la tension singularisante de soi pris dans la dialectique coénonciative et interactionnelle, si l'ethos, c'est la parole incorporée à des fins argumentatives, le style, c'est la recherche de l'affirmation d'une singularisation de la parole dans le cadre du jeu avec les contraintes socio-culturelles des genres, qui s'imposent aux individus. Dans les trois cas, 1'approche moniste devrait aller de soi : l'idiolecte, comme l'ethos, comme le style ne reposent pas sur une sélection arbitraire de traits : c'est l'ensemble du matériau langagier (et des paramètres paraverbaux) qui fait sens, rapporté à des genres, à la dialectique du même et de l'autre ou à des fins argumentatives.

L'intrication des données sociales et individuelles, dans la construction des sujets, est au cœur des diverses conceptions du style, de l'idiolecte et de l'ethos, qui accordent plus ou moins d'importance à telle ou telle donnée et à leur articulation. Ces interrelations nous rappellent que tout individu, dans la singularité de sa construction sociale, n'existe que par autrui et grâce à la collectivité à laquelle il appartient, par les appartenances multiples qui l'aident à construire sa personnalité, ses valeurs, à ajuster ses comportements pratiques et ses représentations. En-

(34) Ainsi que le souligne Eggs 1999 : 44-46, 1'ethos ne peut être considéré comme la dimension fondamentale de l'argumentation que parce qu'il englobe logos et pathos, ou, plus exactement, parce qu'il inclut en lui une dimension spécifique du logos, basée sur le vraisemblable, le raisonnable (phrónesis), distinct du logos de nature inférentielle qui est au cœur du raisonnement logique :

\begin{tabular}{|lll|}
\hline & $\begin{array}{l}\text { Ethos } \\
\text { raisonnable } \\
\text { (phrónesis) }\end{array}$ & $\begin{array}{l}\text { solidaire } \\
\text { (eúnoia) } \\
\text { (logathos) }\end{array}$ \\
\hline
\end{tabular}

NB : Aristote ne place pas la phrónesis dans le logos, parce qu'elle est liée à la situation concrète, et donc elle inclut la personne concrète de l'orateur. D'où la nécessité de dédoubler le logos en logos inférentiel et en logos raisonnable. 
core le mot individu est-il insatisfaisant : car nous visons moins l'être humain à travers ses caractéristiques physiques que la dimension sociale de celui qui existe pour autrui et par autrui, bref, un processus de singularisation / spécification qui nous construit en tant que personne. C'est ce que rappelle Arendt, à partir de l'étymologie du mot persona, dont la dimension sociale et culturelle (à la différence de homo ) a servi de base à la représentation théorique de l'homme social, sujet politique et sujet du droit, en insistant avec beaucoup de pertinence sur le fait que la construction idiosyncrasique des sujets se fait par le biais de rôles divers, qui, de surcroît, permettent d'acquérir la reconnaissance sociale de ses semblables (Arendt, $2005:$ 43-45).

Sans prétendre apporter un éclairage définitif sur les relations entre style, idiolecte et ethos, notre investigation, menée à la lumière de la dialectique du singulier et du social dans la dynamique de la construction de soi, est riche d'enseignements théoriques et pratiques. Sur un plan didactique, si la construction des identités singulières et des subjectivités opère sur le terrain des pratiques sociales et de l'appropriation des normes culturelles, il s'ensuit que l'enseignant de français, qui est aussi un enseignant de lettres, a tout intérêt à exploiter cette dialectique dans son enseignement. Cela signifie concrètement que l'approche stylistique des genres offre un terreau de premier ordre pour la maîtrise des régularités stylistiques des genres seconds (comme des genres premiers), et que c'est sur la base de cette connaissance que le sujet individuel peut faire œuvre de variations individuelles significatives, à l'écrit ${ }^{(35)}$ comme à l'oral. Ce qui est vrai au plan du travail de construction de soi sur le plan du/des style(s) l'est tout autant pour l'idiolecte et l'ethos, puisque les signes et valeurs attachés à notre singularité se renégocient constamment dans les interactions en face à face, selon une visée argumentative qui entre en jeu dans la redéfinition d'une urbanitas moderne.

\section{Références bibliographiques}

Amossy, R. (2006) : L'argumentation dans le discours. $2^{\mathrm{e}}$ édition. Paris : Armand Colin.

Angenot, M. (1982) : La parole pamphlétaire. Paris : Payot.

ARENDT, H. (2005) : Responsabilité et jugement. Paris : Payot.

AuthIER-REVuz, J. (1995) : Ces mots qui ne vont pas de soi. Paris : Larousse.

BAKHTINe, M. (1978 [1975]) : Esthétique et théorie du roman. Paris : Gallimard.

BARTHES, R. (1970) : «L'ancienne rhétorique », Communications 16, 172-229.

(35) Cette problématique a fait 1'objet de plusieurs publications, $c f$. Rabatel 2004, 2005a, 2006a, 2007. Mais bien d'autres activités sont susceptibles de mettre en œuvre cette dialectique, comme on le voit dans plusieurs articles de ce numéro. 
BERNADET, A. (2001) : «L'historicité de l'auteur : une catégorie problématique », in Une histoire de la "fonction-auteur » est-elle possible? N. Jacques-Lefèvre et F. Regard (éds), 13-31. Saint-Etienne : Publications de l'université de Saint-Etienne.

Bonhomme, M. (1998) : Les figures clés du discours. Paris : Editions du Seuil. - (2005) : Pragmatique des figures du discours. Paris : Champion.

Bourdieu, P. (1992) : Les règles de l'art. Paris : Editions du Seuil.

CicÉron, M. T. (1950 et 1956 [-55]) : De l'orateur. Tomes 1, 2 et 3 (trad. E. Courbaud). Paris : Les Belles Lettres.

- (1964 [-46]) : L'Orateur (trad. Albert Yon). Paris : Les Belles Lettres.

COMBE, D. (2002) : «La stylistique des genres » Langue française 135, 33-49.

Comte-S ponville, A. (1988 [1984]) : Le mythe d'Icare. Traité du désespoir et de la béatitude, tome 1. Paris : Presses universitaires de France.

D ANBLON, E. (2006) : La fonction persuasive. Anthropologie du discours rhétorique : origines et actualité, Paris : Armand Colin.

DanON-BoileaU, L. (1982) : Produire le fictif. Paris : Klincksieck.

DÉTRIE, C. (2000) : « La figure, une "parole parlante" au plus près du vécu », $\mathrm{Ca}$ hiers de praxématique $35,141-169$.

- (2005) : «La dynamique idiolectalisante, entre singularisation et réitération » Cahiers de praxématique 44, 51-75.

DÉtrie, C. et Neveu, F. (2005) : «Présentation » Cahiers de praxématique, 44, 7 16.

Dubois, J., Edeline, F., Klinkenberg, J.-M., Minguet, P., Pire, F., Trinon, H. (1970) : Rhétorique générale. Paris : Larousse.

DuPRIEZ, B. (1980 [1977]) : Gradus. Les procédés littéraires. Paris : 10/18.

EGGS, E. (1999) : «Ethos aristotélicien, conviction et pragmatique moderne », in Images de soi dans le discours, R. Amossy (éd), 31-59. Lausanne, Paris : Delachaux et Niestlé.

GADET, F. (2003) : La variation sociale en français. Paris : Ophrys.

Genette, G. (2006) : Bardadrac. Paris : Editions du Seuil.

KlinkenberG, J.-M. (2000) : «L'argumentation dans la figure », Cahiers de praxématique $35,59-86$.

Flahault, F. (2006) : "Be yourself! ». Au-delà de la conception occidentale de l'individu. Paris : Mille et une nuits.

FONTANIER, P. (1968 [1821]) : Les figures du discours. Paris : Flammarion.

Foucault, M. (1966, 2001a) : «Entretien avec Madeleine Chapsal », in Dits et écrits 1, 541-546. Gallimard, Paris.

— $(1969,2001 \mathrm{a})$ : «Qu'est-ce qu'un auteur? », in Dits et écrits 1, 817-849. Gallimard, Paris.

— $(1976,2001 \mathrm{~b})$ : «Questions à Michel Foucault sur la géographie », Dits et écrits 2, 28-40. Gallimard, Paris.

HuCHETTE, J. (2001) : «Y a-t-il un “caractère national” des auteurs ? La problématique du goût dans la critique voltairienne des littératures étrangères », in Une histoire de la "fonction-auteur" est-elle possible? N. Jacques-Lefèvre et F. Regard (éds), 155-167. Saint-Etienne : Publications de l'université de SaintEtienne.

JAUBERT, A. (2005) : «Des styles au style. Genre littéraire et création de valeur », in 
Gouvard, J.-M. (éd.), De la langue au style, 37-50. Lyon : Presses universitaires de Lyon.

LATOUR, B. (2006) : Changer de société $\sim$ Refaire de la sociologie, Paris : Editions de la Découverte.

MAINGUENEAU, D. (1999) : «Ethos, scénographie, incorporation », in Images de soi dans le discours, R. Amossy (éd), 75-100. Lausanne, Paris : Delachaux et Niestlé.

Merleau-Ponty, M. (1960) : Signes. Paris : Gallimard.

Meschonnic, H. (1970) : Pour la poétique 1. Paris : Gallimard.

— (1973) : Pour la poétique 2. Paris : Gallimard.

Molinié, G. (1992) : Dictionnaire de rhétorique. Paris : Le Livre de Poche.

Neveu, F. (2004) : Dictionnaire des sciences du langage. Paris : Armand Colin.

NyCKEES, V. (2000) : «Quelle est la langue des métaphores? » Cahiers de praxématique $35,115-139$.

Philippe, G. (2005) : « Traitement stylistique et traitement idiolectal des singularités langagières », Cahiers de praxématique 44, 77-91.

Quintilien (1975, 1977 et 1979 [96 ?]) : Institution oratoire, T.1, 4 et 6 trad. J. Cousin. Paris : Les Belles Lettres.

RABATEL, A. (2004) : Argumenter en racontant. Bruxelles : De Boeck.

- (2005a) : «La visée des énonciateurs au service du lexique : points de vue, (connaissance et) images du monde, stéréotypie », in F. Grossmann, M.-A. Paveau et G. Petit (éds), Didactique du lexique : langue, cognition, discours, 229-245. Université de Grenoble 3, Grenoble : ELLUG.

- (2005b) : « Idiolecte et re-présentation du discours de l'autre dans le discours d'ego ", Cahiers de praxématique 44, 93-116.

- (2006a) : «La lecture comme activité de construction intersubjective du soi à travers l'approche interactionnelle du style », Lidil 33, 55-77.

- (2006b) : «Genette, les voix du texte et la dialogisation », Recherches linguistiques, 28, 165-188.

- (2006c) : « La dialogisation au cœur du couple polyphonie / dialogisme chez Bakhtine », Revue romane, 41-1, 55-80.

— (2007) : «Style et "théorie des styles". Pour une approche moniste de la notion de "moyens d'expression" ", in Bertrand, O., Charolles, M., François, J., Prévost, S., Schnedecker, C. (éds), Discours, diachronie, stylistique du français, 385-400, Berne : Peter Lang.

RASTIER, F. (1994 « Le problème du style pour la sémantique du texte », in Molinié, G. et Cahné, P. (éds) Qu'est-ce que le style? 263-282. Paris : Presses universitaires de France.

- (2001): Arts et sciences du texte. Paris : Presses Universitaires de France.

RHÉTORIQUE À HERRENNIUS (1989 [-88 ?]) : (trad. Guy Achard) Paris : Les Belles Lettres.

VialA, A. (1985) : La naissance de l'écrivain. Paris : Editions de Minuit.

VouillouX, B. (2005) : «Les prédicats stylistiques », in Gouvard, J.-M. (éd), De la langue au style, 319-355. Lyon : Presses universitaires de Lyon. 